\title{
Evaluation of maternal and neonatal results after immediate labor induction by vaginal misoprostol and expectational treatment
}

\author{
Talaat Dabbaghi Ghaleh ${ }^{1}$, Fatemeh Lalooha ${ }^{1}$, Rashin Normohammadi ${ }^{1}$ and Omid Mashrabi ${ }^{2 \star}$ \\ ${ }^{1}$ Faculty of Medicine, Ghazvin University of Medical Sciences, Ghazvin, Iran. \\ ${ }^{2}$ Faculty of Medicine, Tabriz University of Medical Sciences, Tabriz, Iran.
}

Accepted 5 January, 2012

\begin{abstract}
Annual incidence of induced or strengthened labor in the United States, with nearly doubled increase, has risen from $20 \%$ in 1989 to $38 \%$ in 2002 . For the success of labor induction, cervical status or its desirability is important. Different techniques are used to prepare the cervix. One of these techniques is using misoprostol tablets (Prostaglandin E1 (PGE1)). The aim of this study is to evaluate the maternal and neonatal results after immediate labor induction by vaginal misoprostol and expectational treatment. In a clinical trial conducted in Kosar hospital on 100 patients with diagnosis of premature rupture of the membranes (PROM) in 2006, the maternal and neonatal results that followed the immediate labor induction by vaginal misoprostol and expectational treatment for $12 \mathrm{~h}$ and then induction by oxytocin were investigated. The mean age of women was $23.8 \pm 4.9$ years in misoprostol group and $25.2 \pm 5.6$ years in the expectational treatment group $(P=0.197)$. The mean duration of pregnancy in women was $38.5 \pm 1.2$ weeks in misoprostol group and $38.5 \pm 1.1$ weeks in the expectational treatment group $(P=0.927)$. Mean parity of women was $1.74 \pm 0.98$ in misoprostol group and $1.56 \pm 0.86$ in the expectational treatment group $(P=0.333)$. The mean latency was $9.5 \pm 2.6$ in misoprostol group and $14 \pm 4.3$ expectational treatment group, which in misoprostol group was significantly less than that in the expectational treatment group $(P<0.000)$. The mean interval from peak recruitment contraction was $12.3 \pm 2.9$ in misoprostol group and $17.5 \pm 4.9$ in the expectational treatment group which in misoprostol group was significantly less than in the expectational treatment group $(P<0.000)$. Mean Apgar score in misoprostol group was significantly greater than that in the expectational treatment group $(P=0.02)$. The mean hospitalization duration of misoprostol group was significantly less than that of the expectational treatment group $(P<0.000)$. Frequency of vaginal delivery was $92 \%$ in the misoprostol group and $76 \%$ in the expectational treatment group; and the frequency of cesarean delivery was $8 \%$ in the misoprostol group and $24 \%$ in the expectational treatment group; so, frequency of cesarean delivery in the misoprostol group was significantly lower than that in the expectational treatment group $(P<0.029)$. Whereas labor induction by misoprostol shortens the duration of delivery and requires no long-term hospitalization and reduces costs for patient and hospital, its application is recommended in all low-risk patients. Since misoprostol reduces cesarean sections (CS) level, it is better to be used in low-risk women and this way, the risks of surgery and anesthesia risks related problems would be decreased. Also, regarding the fact that misoprostol, when compared with oxytocin, it administration requires no tight control and no individuals to constantly be on the clinical presence of the patient, it is more economical in terms of employment and need for medical personnel.
\end{abstract}

Key words: Premature rupture of the membranes (PROM), labor induction, misoprostol.

\section{INTRODUCTION}

According to the National Health Statistics Center, the incidence of annual induced or strengthened labor in the
United States has nearly doubled, from $20 \%$ in 1989 to $38 \%$ in 2002 (Cunningham et al., 2008). For a successful 
labor induction, cervical status or its desirability is very important. Different techniques are used to prepare the cervix. One of these techniques is the application of misoprostol tablets (Prostaglandin E1 (PGE1)), which is used in women with gestational age higher than 37 weeks referred with runny. This tablet is administered by finger through the vagina inside posterior fornix with dose of $25 \mu \mathrm{g}$ in $6 \mathrm{~h}$ intervals (Up to maximum $4=100 \mu \mathrm{g}$ ). In women who did not respond to this induction within $24 \mathrm{~h}$, oxytocin IV infusion is administered with the same usual dosage. Administration of misoprostol clearly shortens the incubation period, recruitment and hospitalization time. The aim of this study is to investigate the maternal and neonatal results following an immediate labor induction with vaginal misoprostol and expectational treatment (Lin et al., 2005; Zeteroğlu et al., 2006; Cecatti et al., 2006; Mozurkewich, 2006; Feitosa et al., 2006; Nanda et al., 2007).

\section{MATERIALS AND METHODS}

In a clinical trial conducted in Kosar hospital on 100 patients with diagnosis of PROM in 2006, the maternal and neonatal results following the immediate labor induction by vaginal misoprostol and expectational treatment for $12 \mathrm{~h}$ and then induction by oxytocin were investigated. This study was conducted in Kosar hospital on 100 patients with diagnosis of PROM in 2006, sufficient explanation on how to study, the side effects and its benefits was given to the patients and conscious consent was obtained from them. Diagnosis of PROM was based on clinical history, Speculum examination and in case of necessity, was based on Nitrasin test and Ferm test of vaginal fluid and Ultrasound. After the diagnosis of PROM by physicians, all women underwent biophysical profile (BPP) or cardiotocography (CTG) in order to evaluate fetal status.

\section{Inclusion criteria}

Certain PROM (up to $6 \mathrm{~h}$ after incidence), gestational age equal to or greater than 37 weeks, cephalic presentation and alive fetal showing no sign of distress during the CTG. During the labor, women in each group in terms of temperature, fetal heart rate $(\mathrm{FHR})$ and uterine tone, are controlled. Before entering the labor, both groups of patients had normal BPP and amniotic fluid index (AFI). FHR and fetal status were under close monitoring. $6 \mathrm{~h}$ after the amniotic sac rupture time, similar antibiotics (Ampicillin) was administered in both groups. Hourly vaginal examination was done to assess cervical status. In Misoprostol group, use of tablets was repeated up to 4 doses (100 micrograms), and in case of no response, intravenous oxytocin infusion was administered. In the expectational treatment group, the patient was monitored controlling the temperature and FHR for $12 \mathrm{~h}$, and in case of lack of progress and failure to enter the active phase of labor, intravenous oxytocin was infused. With a simple random sampling method with paper drawing, the patients were placed in either one of the two groups of misoprostol and expectation. Sample size: for the confidence coefficient of $95 \%$, test power of $80 \%$, standard deviation (SD) of $9.8 \mathrm{~h}$, difference of mean of $5.5 \mathrm{~h}$, and 50 patients

*Corresponding author. E-mail: mashrabi1383@yahoo.com. Tel: +98-9144049694 or +98-4226226106. were obtained for each group.

\section{Data analysis method}

After collecting data, results were tested under statistical tables. Chi square test was applied for analysis of qualitative data, Fisher exact test and t-test for quantitative data. Level of significance was considered as $95 \%$.

\section{RESULTS}

The mean age of women was $23.8 \pm 4.9$ years in misoprostol group and $25.2 \pm 5.6$ years in the expectational treatment group $(P=0.197)$. The mean duration of pregnancy in women was $38.5 \pm 1.2$ weeks in misoprostol group and $38.5 \pm 1.1$ weeks in the expectational treatment group $(P=0.927)$. Mean parity of women was $1.74 \pm .98$ and in misoprostol group and 1.56 \pm 0.86 in the expectational treatment group $(P=0.333)$. The mean latency was $9.5 \pm 2.6$ in misoprostol group and $14 \pm 4.3$ expectational treatment group, which in misoprostol group was significantly less than that in the expectational treatment group $(P<0.000)$. The mean interval from peak recruitment contraction was $12.3 \pm 2.9$ in misoprostol group and $17.5 \pm 4.9$ in the expectational treatment group which in misoprostol group was significantly less than in the expectational treatment group $(P<0.000)$. Mean Apgar score was $9 \pm 0.14$ in misoprostol group and $8.7 \pm 0.7$ in the expectational treatment group, which in misoprostol group was significantly greater than that in the expectational treatment group $(P=0.02)$. The mean hospitalization duration was $2.1 \pm 0.33$ days for misoprostol group and $2.9 \pm 0.76$ days for the expectational treatment group, which of misoprostol group was significantly less than that of the expectational treatment group $(P<0.000)$. Frequency of vaginal delivery was $92 \%$ in the misoprostol group and $76 \%$ in the expectational treatment group; and the frequency of cesarean delivery was $8 \%$ in the misoprostol group and $24 \%$ in the expectational treatment group; so, frequency of cesarean delivery in the misoprostol group was significantly lower than that in the expectational treatment group $(P<0.029)$ (Figure 1$)$.

\section{DISCUSSION}

Results show that in the same conditions in terms of maternal age, gestational age and parity between the two groups of misoprostol and expectational treatment, there is a significantly difference in terms of incubation period, recruitment period, cesarean delivery, neonatal Apgar score and maternal stay in hospital.

Hofmeyr et al. (1999), in the Department of Obstetrics and Gynecology, Coronation Hospital and University of the Witwatersrand, South Africa, demonstrated that misoprostol appears to be more effective than 


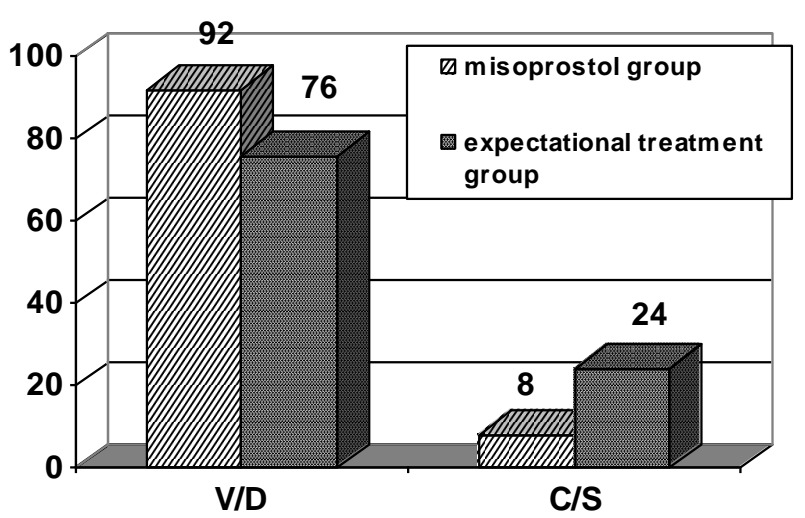

Figure 1. Frequency of delivery type between the two groups

conventional methods of cervical ripening and labor induction. Thus, though misoprostol shows promise as a highly effective, inexpensive and convenient agent for labor induction, it cannot be recommended for routine use at this stage (Hofmeyr et al., 1999).

In a study similar to ours conducted at the Department of Obstetrics and Gynecology Universidade Estadual de Campinas-UNICAMP, Campinas, São Paulo, Brazil, on 150 patients, and the effect of $50 \mu \mathrm{g}$ tablets of misoprostol versus expectational treatment was investigated; they expressed that the average time period from admittance to delivery was significantly shorter in the misoprostol group $(8.68 \pm 4.40 \mathrm{~h})$, which was calculated as $(26.22 \pm 18.98 \mathrm{~h})$ in expectational treatment group $(P=0.001)$ (da Graça et al., 2005).

In a research conducted in Brazil, 150 pregnant women were studied in two groups of immediate induction with misoprostol and expectational treatment, and then induced with oxytocin, the misoprostol group, clearly, had a short incubation period (9.4 versus $15.8 \mathrm{~h})$, shorter induction-to-labor time (18.9 versus $27.5 \mathrm{~h}$ ) and mother hospitalization was also reduced (9).

Wing and Paul (1998) in the Department of Obstetrics and Gynecology, Women's and Children's Hospital, University of Southern California School of Medicine, Los Angeles 90033, USA, demonstrated that vaginal administration of misoprostol (Cytotec) is an effective alternative to oxytocin infusion for labor induction in women with premature rupture of the membranes near term (10).

Farah et al. (1997) in Department of Obstetrics and Gynecology, University of Florida Health Center , Jacksonville, USA demonstrated that although a dose of $50 \mu \mathrm{g}$ is associated with a shorter start-to-delivery interval and a higher incidence of vaginal delivery after one dose. In our study, the latent phase was $4.3 \pm 14$ in the expectational treatment group and $2.6 \pm 9.5$ in misoprostol group. Statistical test indicates significance difference between means of the two groups under study $(P<0.000)$.
In a study by Da Graça et al. (2005), they concluded that the use of misoprostol may reduce the need for oxytocin and increase vaginal delivery within $24 \mathrm{~h}$ after labor induction and lead to reduction of the interval from induction to delivery. Ozden et al. (2002) in Zeynep Kamil Women and Children Education and Research Hospital, Istanbul, Turkey showed that the mean admittancedelivery interval was significantly shorter in the study group $(8.68 \pm 4.40 \mathrm{~h})$ when compared with the control group (26.22 $\pm 18.98 \mathrm{~h}, \mathrm{P}=0.001)$ and the mean interval from membrane rupture to delivery were also significantly shorter in the study group $(19.37 \pm 7.20 \mathrm{~h})$ than the control group $(33.05 \pm 20.85 \mathrm{~h}, \mathrm{P}=0.001)$. Oxytocin necessity was significantly lower in the study group than the control group (45.2\% versus $100 \%, P=0.00051)$ (Ozden et al., 2002).

In our study, the interval from recruitment was $17.5 \pm$ 4.9 in the expectational group and $12.3 \pm 2.9$ in the misoprostol group; which indicates statistically significant difference between the two groups $(P<0.000)$. The mean duration of hospitalization was $2.1 \pm 0.33$ in the misoprostol group and $2.9 \pm 0.76$ in the expectational group and the rate of vaginal and cesarean delivery were, respectively 92 and $8 \%$ in the misoprostol group and 76 and $24 \%$ in the expectational treatment group, which indicates a significant difference between the two groups $(P=0.029)$. Use of misoprostol may reduce the need for oxytocin and increase vaginal delivery rate within $24 \mathrm{~h}$ after labor induction (Alfirevic and Weeks, 2006). It is effective, safe and economic to use misoprostol vaginally in PROM cases with low Bishop Scores at term (Ozden et al., 2002).

\section{Conclusion}

Since labor induction by misoprostol shortens the duration of delivery and requires no long-term hospitallization and reduces costs for patient and hospital, its application is recommended in all low-risk patients.

Since misoprostol reduces cesarean sections (CS) level, it is better to be used in low-risk women and this way, the risks of surgery and anesthesia risks related problems would be decreased. Also, regarding the fact that misoprostol, when compared with oxytocin, requires no tight control and no individuals to constantly be on the clinical presence of the patient when administered, so, it is more economical in terms of employment and need for medical personnel.

\section{REFERENCES}

Cunningham SG, Jant NS, Leveno KJ (2008). Williams Obstetrics, 22 th. Ed. Mc-Graw-hill Company, Philadelphia, pp. 626-636.

Lin MG, Nuthalapaty FS, Carver AR, Case AS, Ramsey PS (2005). Misoprostol for labor induction in women with term premature rupture of membranes: a meta-analysis. Obstet. Gynecol., 106(3): 593-601.

Zeteroğlu S, Engin-Ustün Y, Ustün Y, Güvercinçi M, Sahin G, Kamaci 
M (2006). A prospective randomized study comparing misoprostol and oxytocin for premature rupture of membranes at term. J. Matern. Fetal Neonat. Med., 19(5): 7- 283.

Cecatti JG, Tedesco RP, Pires HM, Calderon IM, Faúndes A (2006). Effectiveness and safety of a new vaginal misoprostol product specifically labeled for cervical ripening and labor induction. Acta Obstet. Gynecol. Scand., 85(6): 11-1706.

Mozurkewich E (2006). Prelabor rupture of membranes at term: induction techniques. Clin. Obstet. Gynecol., 49(3): 83-8672.

Feitosa FE, Sampaio ZS, Alencar CA, Amorim MM, Passini R (2006). Sublingual vs. vaginal misoprostol for induction of labor. Int. J. Gynaecol. Obstet., 94(2): 5-91.

Nanda S, Singhal SR, Papneja A (2007). Induction of labour with intravaginal misoprostol and prostaglandin E2 gel: a comparative study. Trop. Doct., 37(1): 4- 21.

Hofmeyr GJ, Gülmezoglu AM, Alfirevic Z (1999). Misoprostol for induction of labour: a systematic review. Br. J. Obstet Gynaecol., 106(8): 798-803.

da Graça Krupa F, Cecatti JG, de Castro Surita FG, Milanez HM, Parpinelli MA (2005). Misoprostol versus expectant management in premature rupture of membranes at term. , 112(9): 1284-90.
Wing DA, Paul RH (1998). Induction of labor with misoprostol for premature rupture of membranes beyond thirty-six weeks' gestation. Am. J. Obstet. Gynecol., 179(1): 9- 94.

Farah LA, Sanchez-Ramos L, Rosa C, Del Valle GO, Gaudier FL, Delke I, Kaunitz AM (1997). Randomized trial of two doses of the prostaglandin $E 1$ analog misoprostol for labor induction. Am. J. Obstet. Gynecol., 177(2): 9-364.

Ozden S, Delikara MN, Avci A, Fiçicioglu C (2002). Intravaginal misoprostol vs. expectant management in premature rupture of membranes with low Bishop scores at term. Int. J. Gynaecol. Obstet., 77(2): 15-1109.

Alfirevic Z, Weeks A (2006). Oral misoprostol for induction of labour. Cochrane Database Syst. Rev., 19(2): CD001338. 